\section{SURFACE WAVINESS RESULTING FROM SINGLE POINT DIAMOND DRESSING IN CYLINDRICAL GRINDING}

\author{
Jim Redmond, Terry Hinnerichs, Ernest Apodaca \\ Sandia National Laboratories \\ P.O. Box 5800 MS 0439 \\ Albuquerque, NM 87185-0439 \\ Ph: (505) 844-3136 Fax: (505) 844-9297
}

\section{RECEIVED \\ APR 011996 \\ OSTI}

\begin{abstract}
This paper describes an experimental investigation of workpiece surface waviness that stems from poor single point diamond wheel dressing procedures in cylindrical grinding. If done improperly, single point dressing can produce a thread on the grinding wheel surface that is then imposed on the workpiece during machining. The circumferential waviness exhibited by the threaded workpiece is similar to that resulting from one per rev vibrations of the grinding wheel. In order to differentiate between these two sources of waviness, a geometrical approach to predicting the circumferential and axial waves produced during grinding is presented. The concepts presented are illustrated through a series of plunge grinding tests incorporating dressing procedures of varying quality. Test results verify that dressing induced circumferential waviness is similar to waviness resulting from one per rev type vibrations of the grinding wheel. The two sources can be distinguished, however, through examination of the workpiece waviness in the axial direction.
\end{abstract}

\title{
INTRODUCTION
}

Recent advances in sensor and actuator technology have rekindled interest in the monitoring and control of workpiece waviness in cylindrical grinding (Refs. 1-2). Vibration at the tool workpiece interface has long been recognized as a significant source of workpiece waviness. In regenerative chatter, random disturbances initiate a waviness on the workpiece and the grinding wheel that cause the amplitude of the waviness and the grinding forces to steadily increase (Ref. 3-4). Previous vibrations drive current vibrations until the wheel and workpiece intermittently lose contact, emitting the characteristic sound of chatter. Forced vibrations resulting from external influences or internal machine dynamics can also adversely affect the workpiece surface finish. One per revolution vibrations of the grinding wheel caused by imbalance or bearing misalignment have been shown to produce workpiece lobing. In this case the number of lobes produced is generally a multiple of the wheel to workpiece speed ratio (Ref. 1).

In a related phenomenon, the use of single point diamond dressing techniques can also significantly influence the quality of the workpiece. Specifically, a helical thread is introduced into the grinding wheel surface which is then imposed on the workpiece during grinding (Ref. 5-7). Although the prominence of the resulting thread on the workpiece can usually be reduced by reducing the traverse rate and the depth of cut during dressing, some remnant of the dressing procedure will remain on the workpiece. In a circumferential profile of the workpiece, the thread manifests 


\section{DISCLAIMER}

Portions of this document may be illegible in electronic image products. Images are produced from the best available original document. 
itself as waviness that is similar to that produced by one per rev vibrations of the grinding wheel. Therefore, the source of waviness is often difficult to distinguish and the proper corrective action is unclear.

In this paper a geometrical framework for predicting the waviness resulting from single point diamond dressing is presented. The similarity between one per rev vibration induced waviness and dressing induced waviness is discussed, and a technique to distinguish between the two causes is presented. Whereas the one per rev vibration of the grinding wheel produces no discernible waviness in the axial direction, the axial waviness that results from the dressing shows a predictable dependence on the dressing parameters. Therefore, the workpiece axial waviness can be used to pinpoint the cause of the circumferential waviness, and proper corrective action can be initiated. The analysis presented is verified through a series of plunge grinding tests incorporating various dressing procedures.

\section{SINGLE POINT DIAMOND DRESSING}

A schematic diagram of the dressing procedure is given in Figure 1. The grinding wheel has been 'unrolled' such that the entire surface is visible. Furthermore, the dimensions have been distorted to simplify this discussion. In practice, wheel dressing can be somewhat more complicated than the illustration due to alternating positive and negative traverses. This can produce interwoven spiral patterns on the grinding wheel depending on how much material is removed during each pass. Here we consider only a single traverse to elucidate the underlying principles.

The grinding wheel of diameter $d_{g}$ and width $w$ is rotating about the positive $Y$ axis with angular speed $\omega_{g}$ in rev/sec. The single point dressing apparatus is traversing the surface of the grinding wheel along the negative $Y$ axis with speed $T$. For simplicity, it is assumed that the stylus tip is rounded and that the traverse speed is large enough to

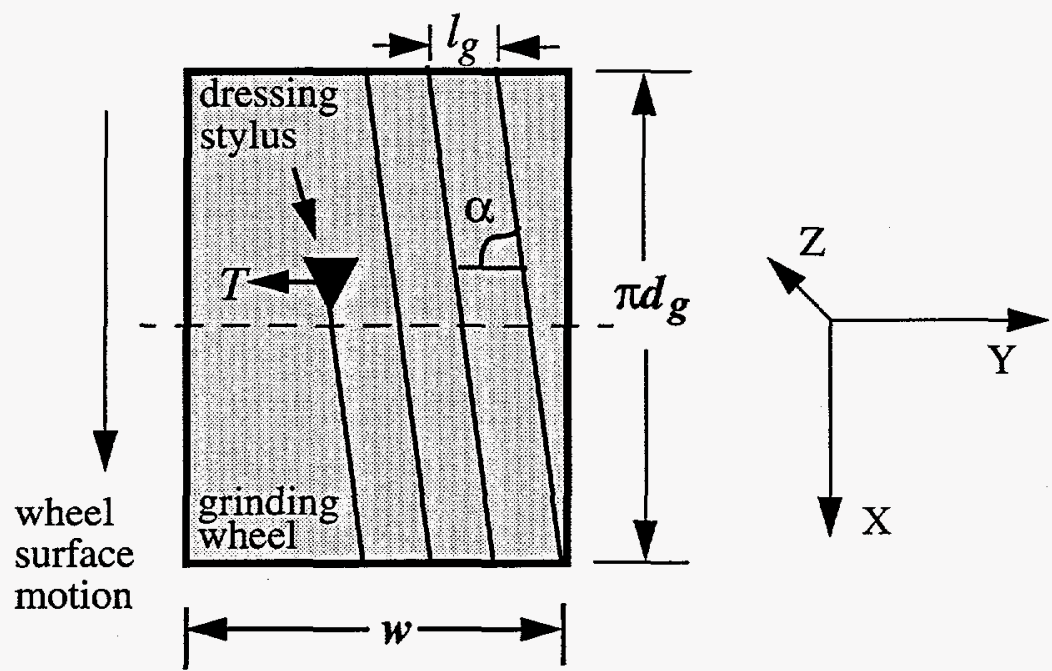

Figure 1. - Diagram of an Unrolled Grinding Wheel During Single

Point Diamond Dressing. 
prevent overlapping cuts. Therefore, the rotation of the grinding wheel combined with the linear motion of the diamond tip produce a spiral pattern on the wheel with angle $\alpha$ defined by

$$
\tan \alpha=\frac{\pi d_{g}}{l_{g}}
$$

where $l_{g}$ is the spiral lead. The lead is the axial travel distance per revolution of the grinding wheel and is given by

$$
l_{g}=\frac{T}{\omega_{g}}
$$

Note that for consistency in units, the lead is given in distance per revolution. This is accommodated in equation 1 by defining the circumference in the same manner. Then substituting Equation 2 into Equation 1, the wheel spiral angle as a function of dressing parameters is given by

$$
\tan \alpha=\frac{\pi d_{g} \omega_{g}}{T}
$$

In practice, the spiral pattern can be made less prominent by decreasing the traverse speed and/or increasing the wheel speed, resulting in a smaller lead. In the limit, $\alpha$ approaches 90 degrees and the spiral pattern diminishes.

\section{GRINDING WHEEL-WORKPIECE INTERACTION}

For the case of a single point stylus with finite traverse speed, the thread imposed on the grinding wheel during dressing can cause undulations on the workpiece during grind. This is because the peaks between the spiral valleys on the grinding wheel represent $N$ individual cutting surfaces given by

$$
N=\frac{w \omega_{g}}{T}
$$

where $N$ is equal to the total number of wheel rotations for a complete traverse during dress. The profile developed during dressing is then imposed on the workpiece as shown in Figure 2. The workpiece has diameter $d_{w}$ and turns with angular speed $\omega_{w}$. The grinding wheel and the workpiece rotate in the same direction, with their relative motion in opposition at the interface. A diagram of an unrolled workpiece during grinding is shown in Figure 3. The grinding wheel essentially plays back the dressing history to the workpiece that was recorded during dressing. Therefore, if the grinding wheel speed remains consistent between dressing and grinding, the $N$ cutting surfaces of the grinding wheel 
move across the workpiece cutting zone with speed $T$. The resulting workpiece spiral angle, however, differs from that of the grinding wheel as a consequence of their differing surface speeds. The resulting spiral angle $\beta$ is given by

$$
\tan \beta=\frac{\pi d_{w}}{l_{w}}
$$

in which $l_{w}$ is the workpiece spiral lead given by

$$
l_{w}=\frac{T}{\omega_{w}}
$$

Substituting equation 6 into equation 5 defines the workpiece spiral angle in terms of the operational parameters as

$$
\tan \beta=\frac{\pi d_{w} \omega_{w}}{T}
$$

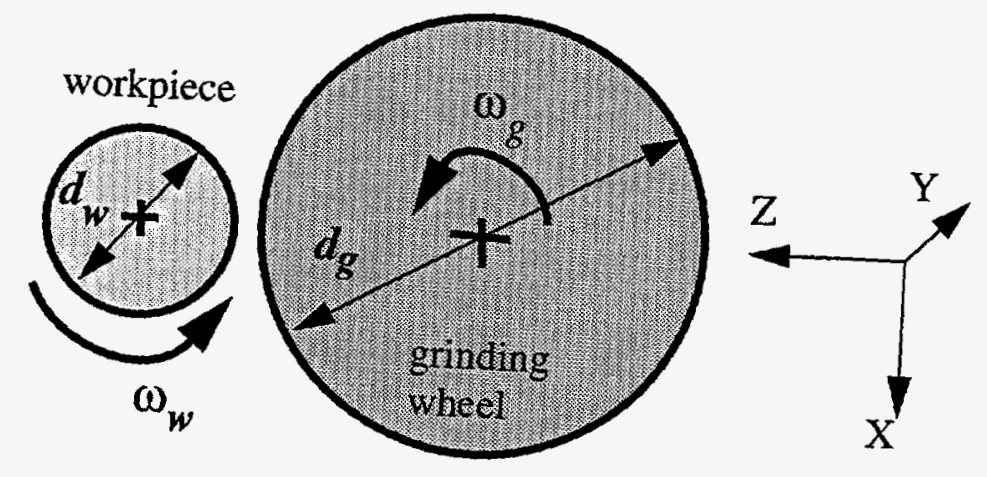

Figure 2. Plunge Grinding Setup Considered for Grinding Wheel Workpiece Interaction.

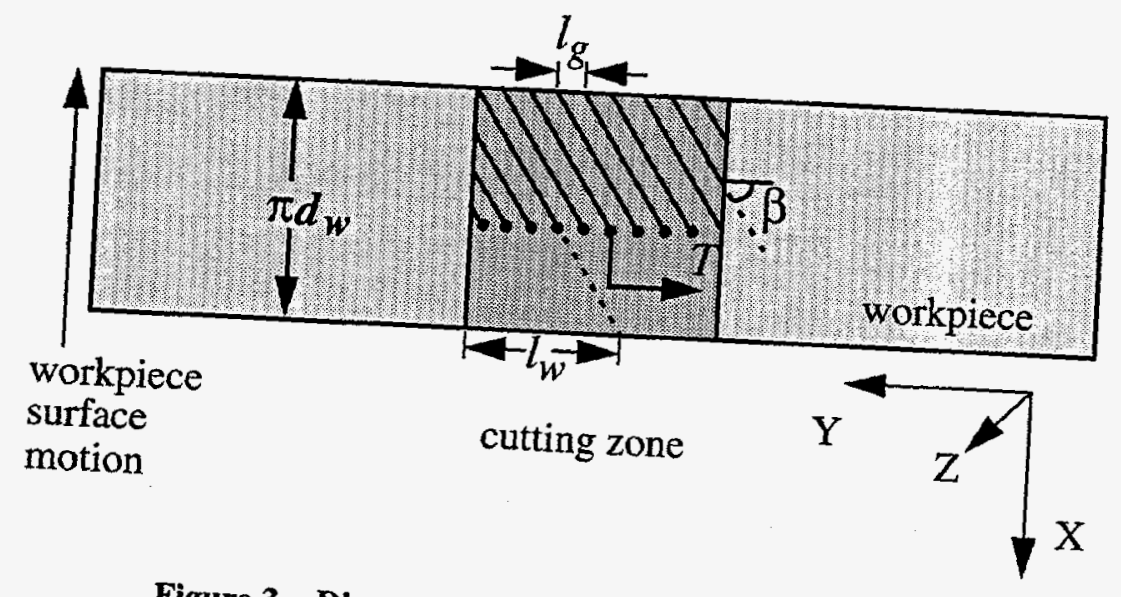

Figure 3. - Diagram of Workpiece During Grinding. 
which is independent of the wheel speed.

\section{Workpiece Circumferential Waviness}

When a circumferential profile of the workpiece surface is produced, spiral patterns manifest themselves as waviness. Whereas the grinding wheel would exhibit only a single wave as a consequence of a bad dressing, the number of oscillations encountered on the circumference of the workpiece depends upon the number of workpiece rotations during which the pattern is recorded. During plunging operations, previous patterns are partially erased with subsequent rotations of the workpiece. If the depth of cut is sufficiently large to completely erase the pattern cut during the previous rotation, the number of oscillations left on the circumference is given by

$$
N_{c 1}=\frac{\pi d_{w}}{l_{g} \tan \beta}
$$

where $N_{c 1}$ is the number of circumferential waves imposed during a single rotation of the workpiece. Rearranging equation 5 and substituting the result into equation 8 yields the number of waves as a ratio of the lead distances

$$
N_{c 1}=\frac{l_{w}}{l_{g}}
$$

Finally, substituting equation 2 and equation 6 into equation 9 yields

$$
N_{c 1}=\frac{\omega_{g}}{\omega_{w}}
$$

From equation 10, the number of waves produced in a single workpiece rotation as a result of a dressing induced spiral depends only on the grinding wheel to workpiece speed ratio.

With sufficiently high plunge rates, a spiral pattern or a one per rev vibration will likely be obscured by the vibration induced at the workpiece-grinding wheel interface. Therefore, it may be difficult to verify the number of cycles predicted by Equation 10 solely by examining the workpiece circumference. When lighter depths of cut are considered, it may require several workpiece rotations to completely eliminate a previously cut spiral pattern or a one per rev vibration pattern. Therefore, a circumferential profile will reveal several superimposed patterns of varying amplitude. Of immediate interest is the case of sparkout in which the amplitudes of the patterns will be relatively consistent since the position of the grinding wheel is held constant throughout the dwell. Depending on the length of the dwell and the speed ratio, perhaps dozens of spiral patterns are superimposed, and the number of circumferential waves encountered on the workpiece will be some multiple of that produced in a single rotation given in Equation 10. The total circumferential oscillations $N_{c}$ is given by 


$$
N_{c}=i \frac{\omega_{g}}{\omega_{w}}
$$

in which $i$ is the integer number of workpiece rotations during which the spiral pattern is recorded. Note that for certain combinations of rotational speed, the spiral patterns eventually overlap and $N_{c}$ becomes an integer. In this case, $i$ is taken as the smallest integer that produces an integer value of $N_{c}$, which then remains constant regardless of subsequent workpiece rotations. Again, the number of circumferential oscillations predicted by equations 10 and 11 are valid for waviness caused by either a one per rev grinding wheel vibration or a dressing induced spiral cut. Therefore, in either case, an effective approach to waviness reduction is to manipulate the speed ratio such that $i$ increases. Although the total number of waves on the surface will increase, the prominence of the waves will decrease.

\section{Workpiece Axial Waviness}

As previously described, the number of circumferential waves predicted by equation 11 is valid for both one per rev vibrations and dressing induced spiral. So in terms of circumferential waviness, the two causes are indistinguishable. To distinguish between these two sources of circumferential waviness, waviness measured axially across the cutting zone must be measured. Since one per rev vibrations of the grinding wheel result in uniform relative displacements between the grinding wheel and workpiece across the entire cutting zone, no discernible axial waviness is introduced. Conversely, the number of axial waves produced on the workpiece as a result of a dressing induced spiral depends on the dressing procedure. For a single revolution of the workpiece, the axial waves produced is equal to $N$, the number of grinding wheel cutting surfaces given in equation 4. For a sparkout workpiece, the total number of axial waves is given by

$$
N_{a}=i \frac{w \omega_{g}}{T}
$$

where $i$ is again the number of workpiece rotations between perfect overlap of the threads given in equation 11. Unlike the circumferential waves, the number of axial waves produced depends on the workpiece speed indirectly through $i$. However, the number of axial waves is heavily dependent on the traverse speed during dress. Intuitively, the number of axial waves decreases for increasing traverse rates. 


\section{EXPERIMENTAL VERIFICATION}

A number of grinding tests were performed to verify the existence of dressing induced waviness on finished workpieces. All tests were performed at Sandia National Labs on a Landis cylindrical grinder using a Norton grinding wheel that was nominally 18 inches in diameter and 1 inch wide. Mild steel workpieces were used for this investigation, and were nominally 2 inches in diameter and 8 inches long. To ensure a regular repeat of the waviness pattern ( $i=1$ ), the workpiece speed was set at $344 \mathrm{rpm}$ which is $1 / 5$ of the fixed grinding wheel speed of $1720 \mathrm{rpm}$. These speeds were consistent throughout all tests considered. Therefore, it was expected that a poor dressing procedure would leave a 5 lobe remnant on the circumference of the workpiece as demonstrated by equation 11 .

The first series of tests were performed to verify that no significant one per revolution vibrations resulting from wheel imbalance or other possible sources were present. Therefore, the wheel was first uniformly dressed using a diamond matrix dressing tool. Then, several workpieces were subjected to a plunge grind with a depth of 0.007 inches, a rate of approximately 0.002 inches/second, and a dwell to sparkout of 8.5 seconds. A representative set of surface profiles for these workpieces is shown in Figure 4. Only a slight 5 lobe waviness is evident on the workpiece circumference accompanied by a dense low amplitude axial waves. Therefore, one per rev vibrations were not significantly impacting the workpiece surface finish.

The second series of cuts was performed after a single diamond point dressing tool was passed over the surface of the grinding wheel with a traverse speed of approximately 0.87 inches/second. This coarse estimate of the traverse speed was obtained by timing the dressing tool as it made 50 passes above the surface of the grinding wheel. After estimating that overshoot at each end of the stroke accounted for $40 \%$ of the total stroke time, the average was

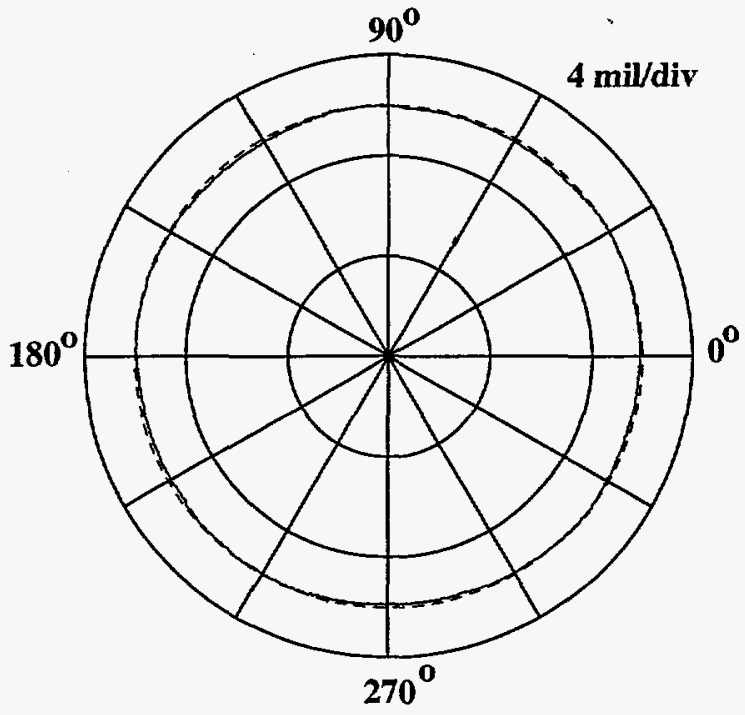

Circumferential Profile

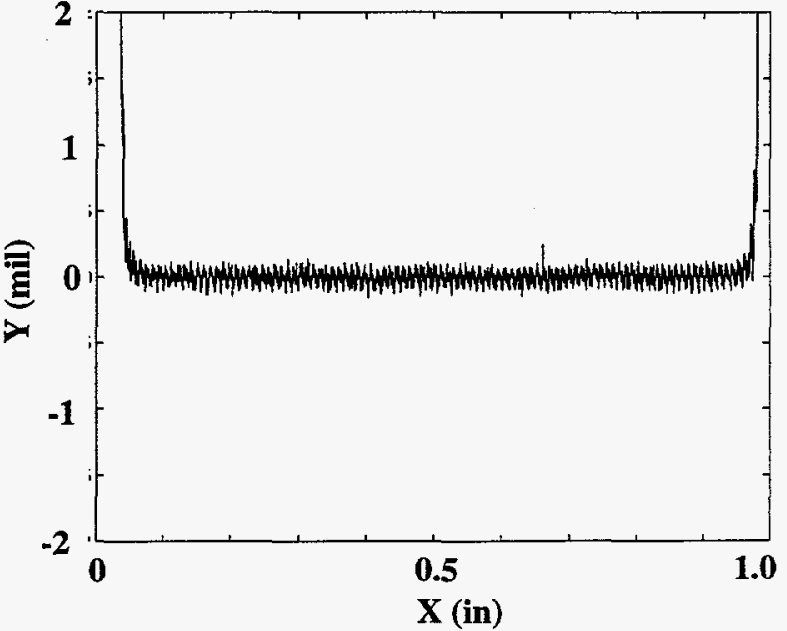

Axial Profile

Figure 4. - Surface Profiles Resulting from a Uniform Dressing Procedure. 
calculated. From equation 2, this traverse rate corresponds to a lead on the grinding wheel of approximately 0.03 inches/revolution. Several plunging cuts with rate, depth, and dwell time comparable to the first series of tests were made and representative profiles are shown in Figure 5. The circumferential profile clearly indicates the presence of 5 lobes on the surface, which is in agreement with equation 10 since the speed ratio was exactly 5 . The axial profile exhibits 29 waves, which is in relative agreement with equation 12 given the uncertainty of the traverse time measured during the dressing procedure. Some 33 axial waves were predicted on the basis of the estimated traverse time. The thread angle was computed from the plots to be 88.4 degrees which compares favorably to 88.6 degrees predicted with equation 7 .

Prior to the next series of tests, the grinding wheel was uniformly dressed and a single plunge to sparkout was completed to verify that the second set of tests did not introduce any significant wheel imbalance that could affect the workpiece surface finish. The resulting surface profiles were similar to those shown in Figure 4, confirming the absence of significant one per rev vibrations. The single point diamond dressing tool was then passed across the surface of the grinding wheel with an estimated traverse speed of 3.125inches/second. Several workpieces were then cut using the same plunging procedure as previously indicated. As in the previous case, five circumferential lobes are evident for a sample workpiece shown in Figure 6. However, in this case the lobes are more pronounced. This stems from the higher traverse rate during dress which lessened the amount of overlap and increased the thread depth on the grinding wheel. The number of lobes present is consistent with equation 11, verifying that the number of lobes resulting from the single point diamond dressing procedure is independent of the traverse rate. In contrast to the previous test series, only 11 axial waves are evident which verifies its dependence on the dressing parameters. Given the uncertainty in the traverse speed, these results are in agreement with the 9.2 axial waves predicted from equation 12 . Based on the

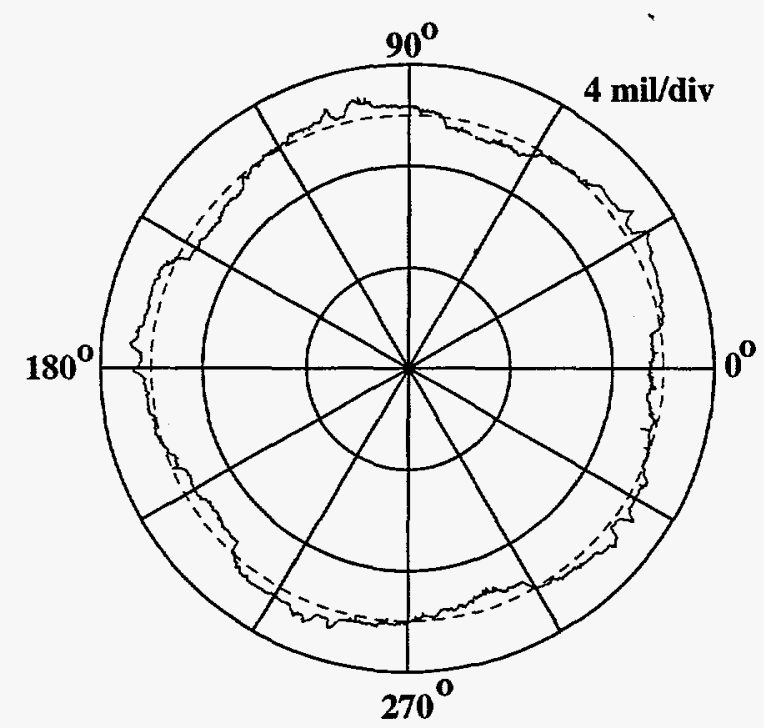

Circumferential Profile

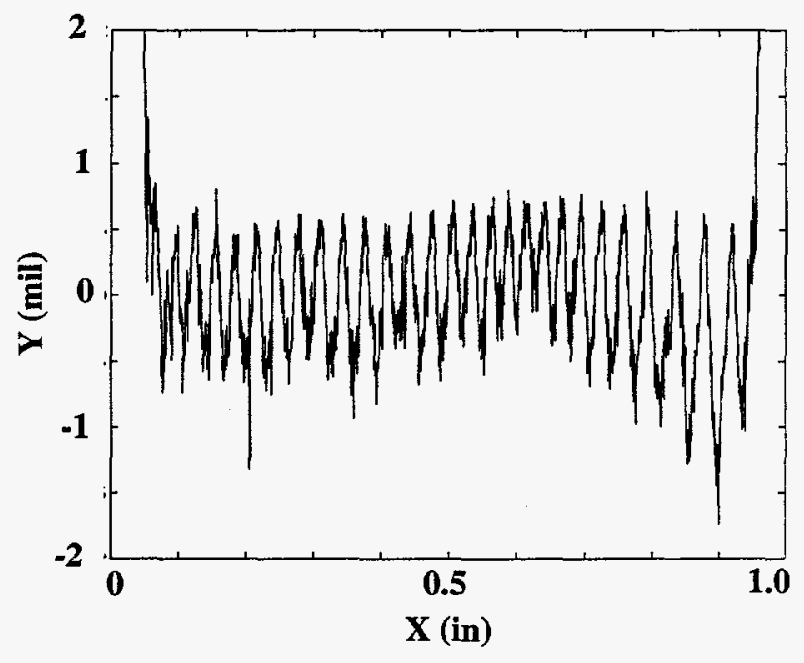

Axial Profile

Figure 5. - Surface Profiles Resulting from a Single Point Diamond Dressing With a Traverse Speed of $0.87 \mathrm{in} / \mathrm{sec}$. 
profiles shown in Figure 6, the estimated workpiece thread angle is given by 85.4 degrees as compared to the 85.0 degrees predicted by equation 7 .

\section{CONCLUSIONS}

This paper described the workpiece waviness characteristics that can result form poor single point diamond wheel dressing procedures in cylindrical grinding. When done improperly, this dressing procedure produces a thread on the grinding wheel which is then imposed on the workpiece during grinding. A geometrical framework was developed to illustrate that dressing induced waviness exhibits some of the same characteristics as waviness resulting from one per rev disturbances of the grinding wheel. In both cases, the number of circumferential waves produced is an integer multiple of the grinding wheel to workpiece angular speed ratio. The two sources can be distinguished, however, by examining the axial waviness. Whereas one per rev vibrations produce no axial waviness, axial waviness resulting from the single point diamond dressing procedure exhibits a predictable dependence on the traverse rate during dressing. This development was then verified through a series of plunge grinding tests completed after subjecting the wheel to dressing procedures of varying quality.

\section{ACKNOWLEDGEMENT}

This work was performed at Sandia National Laboratories, which is operated for the U.S. Department of Energy under Contract No. DE-AC04-94AL85000.

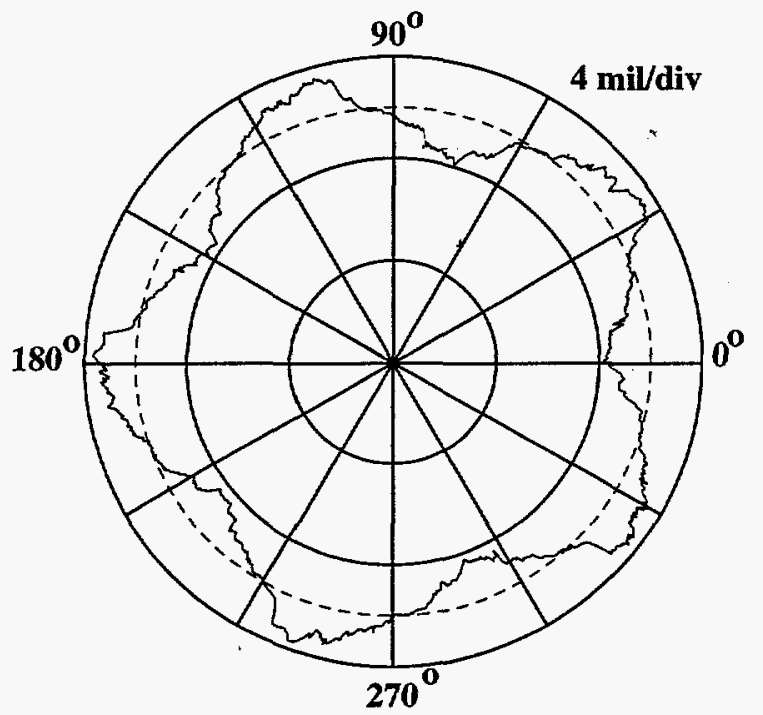

Circumferential Profile

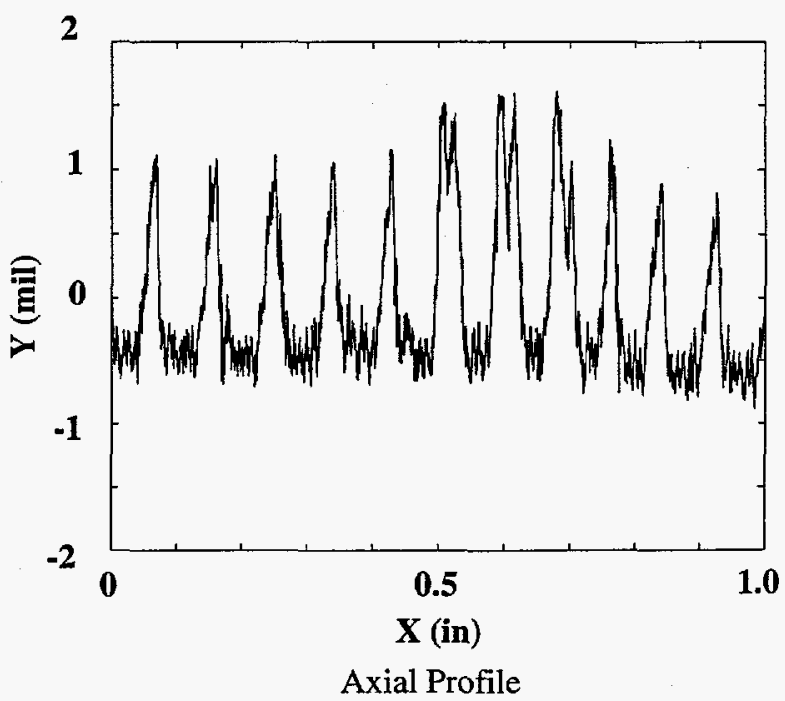

Figure 6. - Surface Profiles Resulting from a Single Point Diamond Dressing With a Traverse Speed of $3.125 \mathrm{in} / \mathrm{sec}$. 


\section{REFERENCES}

1. Tonshoff, H.K., and Foth, M., "In process Detection and Reduction of Workpiece Waviness Caused by Vibrations in External Plunge Grinding," Proceedings of the Third International Grinding Conference, October 4-6, 1988, Fontana, WI, SME Technical Paper \#MR88-620.

2. Weck, E.H.M., Schulze, O., Michels F., and Bonse, R., "Optimization of Machine Tool Performance and Accuracy," Proceedings of the 1994 International Mechanical Engineering Conference and Exposition, November 611, 1994, Chicago, IL, PED-Vol. 68-2, pp. 895-908.

3. Thompson, R.A., "On the Doubly Regenerative Stability of a Grinder: The Theory of Chatter Growth," Journal of Engineering for Industry, Vol. 108, May 1986, pp. 75-82.

4. Kalizer, H., "Analysis of Chatter Vibration in Grinding," Advances in Machine Tool Desgin and Research, Proceedings of the 11th International M.T.D.R. Conference, September 1970, pp. 615-631.

5. Vickerstaff, T.J., Diamond Dressing - Its Effect on Work Surface Roughness," Industrial Diamond Review, July 1970, pp. 260-267.

6. Hahn, R.S. and Lindsay, R.P., "Principles of Ginding...Part V: Grinding Chatter," Machinery, November 1971, pp. 34-40.

7. Lacey, S.J., "Vibration Monitoring of the Internal Centerless Grinding Process, Part 1: Mathematical Models," Proc. Instn. Mech. Engrs, Vol. 204, Part B: Journal of Engineering Manufacture, pp. 119-128, 1990.

\section{DISCLAIMER}

This report was prepared as an account of work sponsored by an agency of the United States Government. Neither the United States Government nor any agency thereof, nor any of their employees, makes any warranty, express or implied, or assumes any legal liability or responsibility for the accuracy, completeness, or usefulness of any information, apparatus, product, or process disclosed, or represents that its use would not infringe privately owned rights. Reference herein to any specific commercial product, process, or service by trade name, trademark, manufacturer, or otherwise does not necessarily constitute or imply its endorsement, recommendation, or favoring by the United States Government or any agency thereof. The views and opinions of authors expressed herein do not necessarily state or reflect those of the United States Government or any agency thereof. 
$-11-$ 